rung der Dosis, also eine Volldosierung bzw. eine Wiederaufnahme der ursprünglich erfolgreichen Therapie wieder rasch zu einer stabilen Remission. „Dies zeigen alle zu diesem Thema durchgeführten Studien“, so Krüger. Bisher habe keine einzige Studie gezeigt, dass eine Therapiereduktion das langfristige Outcome verschlechtert. Allerdings liegen bisher nur vereinzelte Langzeitbeobachtungen nach Therapieabbau vor.

In der RETRO-Studie wurden die für einen Therapieabbau geeigneten Patienten in drei Gruppen randomisiert: Unverändertes Fortführen oder Halbierung oder vollständiges Absetzen der Therapie. Innerhalb eines Jahres wurden folgende Flare-Raten beobachtet: $15,8 \%$ vs. $38,9 \%$ vs. $51,9 \%$. Bei Patienten mit nach- weisbaren Anti-ccP-Antikörpern war die Flare-Rate mit 40\% doppelt so hoch wie bei Anti-ccP-negativen Patienten. Wurde die Therapie dann wieder fortgeführt, so zeigten die Patienten wiederum ein ausgezeichnetes Ansprechen. „Doch sollte man bei einer Therapiereduzierung immer bedenken, dass gerade bei Biologika dies ein Abweichen von der festgelegten Dosierung und somit eine Off-Label-Therapie bedeutet", merkte Krüger an.

\section{Glukokortikoide: Beherzt beginnen, beherzt beenden}

Glukokortikoide sind und bleiben auch im Zeitalter der modernen Biologika unverzichtbar. Sie gelten weiterhin als initiale Standardtherapie in Kombination mit Methotrexat. In Abhängigkeit vom klinischen Bild wird eine Dosis von 10$30 \mathrm{mg}$ täglich empfohlen. Diese Startdosis sollte aber innerhalb von 8 Wochen in den Low-Dose-Bereich $\leq 7,5 \mathrm{mg}$ gesenkt werden, und nach 3-6 Monaten sollte die systemische GlukokortikoidTherapie ganz beendet werden, und zwar wegen des erhöhten Risikos für Osteoporose, Katarakt, Infektionen und KHK. Wenn eine Dauergabe notwendig sein sollte, dann ist eine Dosis von $<5 \mathrm{mg}$ das Minimalziel. Sinnvoll sind evtl. zusätzliche intraartikuläre Gaben eines Glukokortikoids in Gelenke mit hoher Entzündungsaktivität.

Dr. Peter Stiefelhagen

- 45. Kongress der Deutschen Gesellschaft für Rheumatologie (DGRh), Stuttgart, 6.-9.9.2017

\title{
Axiale Spondyloarthritis
}

\section{Nicht jeder Rückenschmerz ist harmlos}

\begin{abstract}
Anhaltende Rückenschmerzen sind häufig, meist aber unspezifisch. Doch wenn diese länger als drei Monate anhalten, sollte man an eine entzündliche Ursache, genauer gesagt an eine axiale Spondyloarthritis denken. Auch hier gilt: Je früher die Therapie beginnt, desto erfolgreicher ist sie.
\end{abstract}

Für einen entzündlichen Rückenschmerz sprechen nach Dr. Uta Kiltz, Herne, folgende Kriterien:

- Langsamer Beginn,

- Morgensteifigkeit in der Wirbelsäule,

- Besserung bei Bewegung, aber nicht in Ruhe,

- Nächtliche Schmerzen, meist in der zweiten Nachthälfte und Besserung durch Aufstehen,

- alternierender Gesäßschmerz,

- Auftreten vor dem 45. Lebensjahr.

\section{Leitliniengerechte Therapie}

Für die Therapie der Spondylarthritiden wurden die Leitlinien vor Kurzem aktualisiert. Danach wird als optimales Ma-

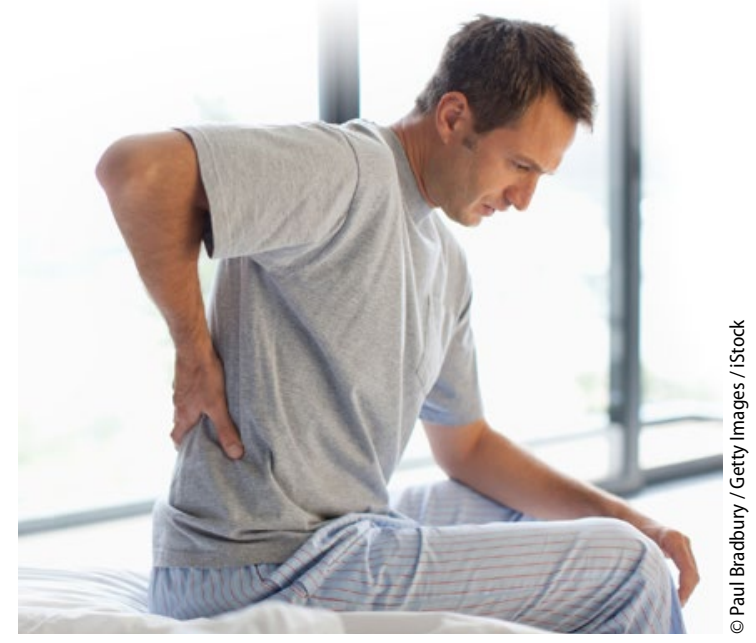

Fragen Sie nach Morgensteifigkeit!

nagement eine Kombination von medikamentösen und nicht-medikamentösen Maßnahmen empfohlen. NSAR sind die First-Line-Medikamente. Sie sollten, soweit erforderlich und verträglich, bis zur maximalen Dosis eingesetzt werden.
Bevor die Therapie eskaliert wird, sollten zumindest zwei NSAR über vier Wochen erprobt sein.

Eine systemische Langzeittherapie mit Glukokortikoiden wird bei rein axialer Erkrankung ebenso wenig empfohlen wie eine konventionelle DMARD(disease-modifying-antirheumatic-drugs)-Therapie. Biologika sollten bei trotz konventioneller Therapie hoher Krankheitsaktivität zum Einsatz kommen, wobei sich initial ein TNFalpha-Inhibitor empfiehlt. Bei Versagen eines solchen kann ein anderer TNFalpha-Inhibitor oder ein Interleukin17-Inhibitor wie Secukinumab eingesetzt werden.

- Dr. Peter Stiefelhagen

- 45. Kongress der Deutschen Gesellschaft für Rheumatologie (DGRh), Stuttgart, 6.-9.9.2017 